\title{
One Case of Forensic Clinical Identification of Ulnar Radius Fracture Not Involving Epiphysis
}

\author{
Dawei Jiang $^{1}$, Yujun Zhou ${ }^{2 *}$ \\ ${ }_{2}^{1}$ Fujian Hengzheng Forensic Laboratory, Sanming 365000, China \\ ${ }^{2}$ Fujian Chengzheng Judicial Identification Center, Zhangzhou 363000, China \\ Email: 839475672@qq.com
}

\begin{abstract}
In order to investigate the relationship between injury and injury of the identified person, to determine the real disability of the identified person, to determine whether the fracture of ulna and radius of the identified person is involved in the epiphysis, and to be commissioned by the court, the identified person is specially re-identified. According to the contents and methods of the Technical Specification for Forensic Identification (SF/ZJD0103003-2011) of the Ministry of Justice, the forensic clinical examination was conducted. After consulting the case data and conducting the forensic clinical examination of the identified person, this appraisal concluded that the left ulna and radius broken line of the patient disappeared. The epiphyseal plate was clear, and there was no deformity. The disability grade was not constructed according to the provisions of the classification of the degree of disability caused by human injury.
\end{abstract}

Keywords: forensic clinical, fracture, epiphyseal, reappraisal

\section{Cases}

\subsection{Summary of cases}

On september 1, 2017, Mr. Wei was taken to the city's first people's hospital for treatment when he was injured by a motorcycle on the side of the road.Afterwards ,Mr. Wei and his family unilaterally commissioned a forensic appraisal institute to carry on the appraisal, the first appraisal is $\mathrm{X}$ grade disability.The defendant , $\mathrm{Mr} \mathrm{Li}$, was not satisfied with the appraisal. At present by the county people's court commissioned to request the expert Mr. Wei disability grade to make a new judicial appraisal.

\subsection{Summary of medical records}

Municipal first people's Hospital outpatient medical records: September 1, 2017: motorcycle crash caused left forearm, wrist pain activities can not be completed. PE left forearm distal ulna and radius swelling, deformity, dyskinesia.1 September 2017, the first people's hospital left ulnar and radial anterior and lateral X (No. 201709010699) showed: fracture of the distal ulnar and radial bone. Diagnosis: fracture of left ulna and radius.

\subsection{Forensic clinical examination}

Female children, mind, spirit can, normal development, good nutrition, physical examination cooperation.

No abnormal head and face, no abnormal neck, no deformity of thoracic symmetry, no tenderness and percussion pain. The abdomen was not abnormal, the physiological curvature of the spine was present, the muscle strength of the left upper limb was normal, the movement range of the left shoulder joint, elbow joint and wrist joint was normal, the muscle strength and muscle tension of the other limbs were normal, and the joint movement was normal.

Film reading: September 1, 2017, the first people's Hospital left ulna and radius lateral X (No. 201709010699) showed: left distal radius fracture, distal fracture to the dorsal displacement, fracture line did not pass through the epiphyseal plate; left ulnar branch fracture.

March 25, 2019 first people's hospital left wrist positive lateral position film (No. 201903250725) shows: left ulna and radius broken line disappeared, epiphyseal plate clear, no malformation. 


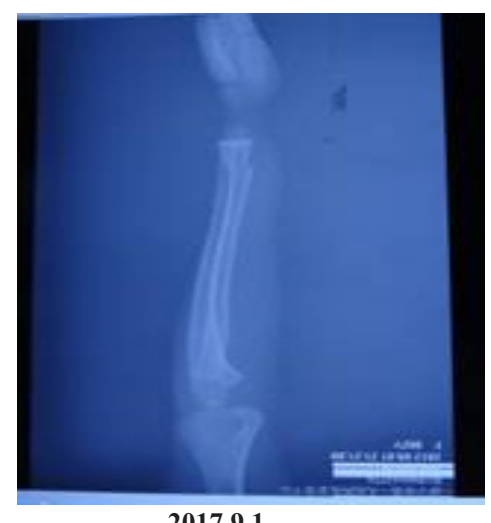

2017.9.1

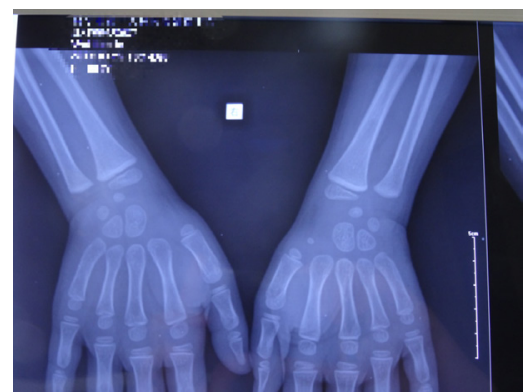

2019.3.5

\section{Discussion}

\subsection{The epiphyseal plate is located between the epiphyseal and metaphyseal ends and is the site of epiphyseal growth and development}

As a component of hyaline cartilage in infancy, called epiphyseal cartilage, epiphyseal chondrocytes continue to divide, proliferate and ossify, making bone grow. Adult, epiphyseal cartilage ossification, and the backbone into one, the remaining traces called epiphyseal plate, epiphyseal formation of articular cartilage, life-long non-osteogeny. Mechanical injury is a common physical factor causing fractures. The healing process of fracture caused by injury is a complex and continuous process, which is divided into the mechanical stage of hematoma inflammation, the original callus formation stage, and the callus transformation molding stage from the histological and cytological changes. Epiphyseal cartilage injury will lead to the formation of a bony link between the epiphyseal and metaphyseal ends in children, that is, bone bridge, so that all or part of the epiphyseal plate is closed early, resulting in the growth of bone dysplasia and limb shortening or angular malformation. Adult long epiphyseal injury will not affect the growth and development of bone ${ }^{[1]}$.

\subsection{Epiphyseal piate fracture is an injury occurring in the epiphyseal part of the backbone or} epiphyseal plate before healing

Damage occurs because the strength of the epiphyseal plate in children is far less than that of the joint capsule and ligament, and before the violence is insufficient to cause ligament and joint capsule damage, it can exceed the limit that the epiphyseal plate can bear to cause Epiphyseal piate fracture.. Clinical manifestations of Epiphyseal piate fracture are local swelling, pain, especially tenderness at the epiphyseal site, and sometimes touching the fracture mass. ${ }^{[2]}$ Therefore, the healing process and outcome of epiphyseal separation or fracture injury are not only related to physical factors, but also to age factors. In childhood, the degree of ossification of epiphyseal cartilage directly affects the growth and development of bone.

\subsection{The identification of Epiphyseal piate fracture is mainly based on the history of trauma,} clinical manifestations and imaging examination after injury

$\mathrm{X}$ line examination mainly by the epiphyseal and metaphyseal pair to judge the relationship.

The diagnosis of trauma history is clear. combined with X line film, CT MRI and other imaging examination judgment.

The degree of ossification of epiphyseal cartilage after injury. Epiphyseal injury in childhood, whether affect 
epiphyseal cartilage ossification, must be identified more than half a year after injury.

\section{Clinical performance}

To analyze whether epiphyseal cartilage and epiphyseal are affected during development, if so, joint deformity or long bone shortening changes occur ${ }^{[3]}$.

\subsection{Findings}

According to the above examination and combined with the medical records, the identified person Wei Tianle left ulna and radius fracture, the left upper limb muscle strength is normal, the left shoulder joint, elbow joint, wrist joint movement range is normal. Read the X film taken at the time of his injury (No. 201709010699) showed that the fracture of the left ulna and radius did not involve epiphyseal, there were no imaging signs of epiphyseal fracture; 1 year and 7 months after the injury X film (No. 201903250725): the fracture line of the left ulna and radius disappeared, the epiphyseal plate was clear and no deformity. Non-disability rating in accordance with the Classification of Disability and Injury ${ }^{[4]}$.

\section{Experience and shortcomings}

For the identification of Epiphyseal piate fracture, first of all, the age of the identified person needs to be judged, epiphyseal cartilage only exists in the juvenile population, adult epiphyseal cartilage ossification, fracture will not accumulate epiphyseal cartilage. Secondly, it is necessary to read the relevant materials of medical records in detail, especially the reading of imaging materials, to clarify the relationship between injury and disease, and the severity of injury. Epiphyseal piate fracture imaging film is not easy to read, need certain experience, need to consult clinical experts if necessary. Finally, for the consequences of injury, it is necessary to judge whether epiphyseal cartilage is affected, and to evaluate the occurrence and possible consequences.

Re-appraisal is a challenging task, especially for the conclusion before negation, which needs to be supported by sufficient factual and theoretical basis to achieve scientific identification. In addition, the assessment of demotion or recognition as not constituting a disability grade requires communication with the family members of the expert.

\section{References}

[1] Cypress Order. Anatomy of Systems. People's Health Publishing House; 2008.

[2] Liu Jihui. Forensic Clinical Edition 5. Beijing: People's Health Publishing House; 2016.

[3] Criteria for determining the degree of human injury. Beijing: Ministry of Justice; 2014.

[4] Du Chengzhu. Forensic identification of epiphyseal injury. Chinese forensic society forensic clinical professional committee. Proceedings of the 17th National Symposium on Forensic Clinical Science, Chinese Forensic Society. Chinese Forensic Society Forensic Clinical Committee: Chinese Forensic Society. 2014; 194-195. 